\title{
Gold nanorings synthesized via a stress-driven collapse and etching mechanism
}

\author{
Jixiang Fang ${ }^{1}$, Jiang $\mathrm{Li}^{2}$, Cuifeng Tian ${ }^{3}$, Qiangqiang $\mathrm{Gao}^{2}$, Xiangjie Wang ${ }^{4}$, Nengyue Gao ${ }^{5}$, Xinglin Wen ${ }^{6}$, \\ Chuansheng $\mathrm{Ma}^{1}$, Hongjun You ${ }^{1,2}$, Zhilin Yang ${ }^{4}$, Qing-Hua $\mathrm{Xu}^{5}$, Qihua Xiong ${ }^{6}$ and Zhiyuan $\mathrm{Li}^{7}$
}

Toroidal Au or Ag nanostructures are of particular interest due to their unique optical responses and superior catalytic applications. However, the fabrication of ring-like $\mathrm{Au}$ or $\mathrm{Ag}$ nanostructures is limited to either electron beam lithography or template techniques, thus hampering their applications. Here, we present a new stress-driven structure collapse and etching mechanism to synthesize Au nanorings via a direct one-pot solution-based chemical reaction. The nanoparticle-mediated recrystallization process contributes to the formation of Au nanoframes, which contain unusual stress, thus promoting the breakup of the nanoframes and finally converting them into Au nanorings. The Au nanorings with tunable hole sizes exhibit interesting localized surface plasmon features owing to the coupling of bonding and antibonding modes on the inner and outer surfaces of the nanorings. This facile approach may open the door for the preparation of toroidal nanostructures in other compositions for numerous applications.

NPG Asia Materials (2016) 8, e323; doi:10.1038/am.2016.163; published online 4 November 2016

\section{INTRODUCTION}

Tailoring the shape-dependent properties of nanoparticles (NPs) has been the focus of intensive research over the past decade owing to their strong shape effects in many fields, including catalysis, electronics, photonics, information storage, optoelectronics and biological labeling. ${ }^{1}$ Among the wide variety of NPs with diverse shapes, 'toroidal/ring' NPs, as artificial complex nanostructures with ring cavities, are of particular interest because they exhibit novel and unique properties in sensing, ${ }^{2}$ magnetic configuration, ${ }^{3}$ optical response ${ }^{4}$ and plasmonic behavior. ${ }^{5}$ In particular, Au or Ag nanostructures with toroidal shapes (nanorings) exhibit near infrared resonances that are not observed in similar disk-like particles without holes. ${ }^{6} \mathrm{Au}$ or Ag nanorings also exhibit substantial increases in bulk refractive index sensitivity relative to those of nanodisks with similar diameters, ${ }^{7}$ remarkably uniform field intensity enhancements for surface-enhanced Raman scattering, ${ }^{8}$ enhanced second harmonic generation at near infrared frequencies ${ }^{9}$ and superior catalytic activities. $^{10}$

To date, Au or Ag NPs with various shapes, such as nanospheres, nanobars, nanorods, nanorices, nanoneedles, nanowires, nanocubes, nanoprisms, bipyramids, nanocarrots, nanoplates, nanodisks and nanobelts, have been successfully prepared. ${ }^{11}$ However, the fabrication of ring-like Au or Ag nanostructures is limited to either electron beam lithography or template techniques. ${ }^{12}$ The electron beam lithography approach not only involves costly and complex processes but also leads to nanorings anchored onto solid supports, which limits their feasibility for further applications. The template method, for example, via a galvanic reaction using a plate-like structure as a template, is an effective route to synthesizing $\mathrm{Au}$ nanoframes or hollow nanostructures; however, it remains technically difficult to obtain completely pure $\mathrm{Au}$ nanostructures without other components. Importantly, this method lacks the ability to produce flexible nanostructures with tunable properties, for example, controllable central hole size. Thus, a straightforward and robust synthesis protocol to synthesize $\mathrm{Au}$ or $\mathrm{Ag}$ nanorings, particularly via direct growth in solution, remains a great challenge.

Different formation mechanisms have been proposed to synthesize 'toroidal/ring' nanostructures with a variety of compositions. For instance, an etching mechanism based on the Ostwald ripening process from the inner side toward the outside has been used to prepare $\mathrm{ZnO}, \mathrm{Fe}_{2} \mathrm{O}_{3}$ and $\mathrm{Bi}_{2} \mathrm{Te}_{3}$ nanorings, as well as $\mathrm{CaCO}_{3}$ microrings. ${ }^{13-16}$ In this case, a higher density of crystallographic defects such as dislocations or linear defects in the center of the disk results in a higher local reaction/etching rate, which consequently leads to the formation of a ring-like structure. A self-rolling or polymer-induced coiling mechanism has been observed to explain the formation of $\mathrm{ZnO}$ nanorings, $\mathrm{Au}$ and $\mathrm{Ag}$ nanosprings, InS rings and loops and $\mathrm{V}_{3} \mathrm{O}_{7}$ nanoscrolls. ${ }^{17-20}$ In addition, a structural

\footnotetext{
${ }^{1}$ School of Electronic and Information Engineering, Xi'an Jiaotong University, Shann Xi, China; ${ }^{2}$ School of Science, State Key Laboratory for Mechanical Behavior of Materials, Xi'an Jiaotong University, Shann Xi, China; ${ }^{3}$ School of Physics and Electronic Science, Shanxi Datong University, Shanxi, China; ${ }^{4}$ Department of Physics, School of Physics and Mechanical Engineering, Xiamen University, Xiamen, China; ${ }^{5}$ Department of Chemistry, National University of Singapore, Singapore, Singapore; ${ }^{6}$ Division of Physics and Applied Physics, School of Physical and Mathematical Sciences, Nanyang Technological University, Singapore, Singapore and 'Institute of Physics, Chinese Academy of Sciences, Beijing, China

Correspondence: Professor J Fang, School of Electronic and Information Engineering, Xi'an Jiaotong University, Xian Ning West Road, 28\#, Xi'an, Shann Xi 710049, China. E-mail: jxfang@mail.xjtu.edu.cn

Received 1 May 2016; revised 28 June 2016; accepted 2 August 2016
} 
transformation via an edge-selective reaction of two-dimensional (2D) layered nanocrystals or via a Kirkendall effect has been used to prepare $\mathrm{TiO}_{2}$ toroids, $\mathrm{Cu}_{2} \mathrm{~S}$ nanorings and $\mathrm{Cu}-\mathrm{NaInS}{ }_{2}$ nanorings. ${ }^{21-23}$ Recently, the self-assembly mechanism of NPs with intrinsic polygons led to the growth of ring-like $\mathrm{CdS}, \mathrm{Ni}-\mathrm{Co}$ and PbSe nanorings. ${ }^{24-26}$ However, until now, it seems that these growth mechanisms have not been observed in the synthesis of ring-like Au or Ag nanostructures, particularly in direct solution-based growth.

Previous investigations of $\mathrm{Au} / \mathrm{DNA}$ ring-like compounds synthesized using DNA as a template seem to indicate that a soft-template protocol could be effective in the construction of $\mathrm{Au}$ ring-like nanostructures. ${ }^{27,28}$ Here, to the best of our knowledge, we report for the first time a novel and simple strategy for the synthesis of Au nanorings via a one-pot direct solution-based chemical reaction by means of an unusual stress-driven structure collapse and etching mechanism. The as-formed Au nanorings with tailored structures are an exciting new member of the family of Au nanostructures with diverse shapes. In particular, the simple addition of sodium dodecyl sulfate (SDS) into the polyethylene glycol (PEG) reaction system enabled the synthesis of Au nanorings with precisely tunable central hole sizes, that is, from approximately hundreds down to approximately tens of nanometers, which is impossible to obtain from previously described methods, for example, galvanic reaction routes. The Au nanorings exhibit interesting hole-size-dependent optical features owing to the coupling of bonding and antibonding modes on the inner and outer surfaces of the nanorings.

\section{MATERIALS AND METHODS}

\section{Materials}

All chemicals were analytically pure and used without further purification. The SDS was purchased from Tianli Reagents Ltd, Tianjin, China. The PEG (average $\mathrm{Mn}=20000)$ and hydrogen tetrachloroauric acid $\left(\mathrm{HAuCl}_{4} \bullet 4 \mathrm{H}_{2} \mathrm{O}, \geqslant 99.9 \%\right)$ were purchased from Shanghai Aladdin Biological Technology Ltd. The water used was highly purified with resistivity $\geqslant 18.0 \mathrm{M} \Omega \bullet \mathrm{cm}$.

\section{Synthesis}

For the synthesis of the Au nanodisks with collapsed centers (reaction-1), $0.6 \mathrm{~g}$ PEG was dissolved with deionized water $(15 \mathrm{ml})$ in a glass vial $(40 \mathrm{ml})$. Then, the vial was placed into a water bath at $\sim 80^{\circ} \mathrm{C}$ under magnetic stirring at 200 r.p.m. After $40 \mathrm{~min}$, the vial was removed to room temperature. When the temperature of the solution decreased to room temperature, $15 \mathrm{ml}$ of a $1 \mathrm{mM}$ $\mathrm{HAuCl}_{4}$ aqueous solution was quickly poured into the solution under strong magnetic stirring. Two minutes later, the vial was placed into a water bath at $\sim 50{ }^{\circ} \mathrm{C}$ for various reaction periods. Then, the upper solution layer was poured out, and the product was obtained. Finally, the samples were washed with deionized water and absolute ethanol for subsequent characterization.

In this study, we also investigated reaction- $2^{29}$ for the synthesis of Au nanorings with tunable central pore sizes. Similar to reaction-1, $0.026 \mathrm{~g}$ SDS and $0.6 \mathrm{~g}$ PEG were dissolved in deionized water $(15 \mathrm{ml})$ in a glass vial $(40 \mathrm{ml})$, and the reaction process was the same as in the synthesis of Au nanoplates with collapsed centers.

\section{Characterization}

The morphologies and structures of the products were characterized using a scanning electron microscope (SEM, JEOL, JSM-7000F) and a transmission electron microscope (transmission electron microscopy (TEM), JEOL, JEM-2100 with an accelerating voltage of $200 \mathrm{kV}$ ). Samples for SEM and TEM observations were prepared on $\mathrm{n}-\mathrm{Si}$ (100) slides and carbon-coated copper grids. The powder X-ray diffraction (XRD) measurements were performed using an XRD diffractometer (D8-Advance, Bruker, Germany) using $\mathrm{Cu} \mathrm{K} \alpha$ radiation $(\lambda=1.5406 \AA)$. Atomic force microscopy (NT-MDT) was used to study the heights of the Au nanoplates and nanorings.

\section{Scattering spectra}

Single-particle scattering measurements were performed using an inverted microscope (Nikon Eclipse Ti). For single-particle scattering spectra measurements, a quartz-tungsten-halogen lamp $(100 \mathrm{~W})$ was utilized as a white light source. A sheet polarizer was utilized to adjust the polarization of the white light. The white light was partially blocked and then focused onto the sample by a dark-field condenser (Nikon, NA $=0.80-0.95$ ). A precise three-dimensional piezoelectric translational stage (PI E-710) was utilized to move the particle of interest into the detecting area. Scattering light from the particle of interest was collected using an oil-immersion objective (Nikon, $\times 100, \mathrm{NA}=0.5$ ) and
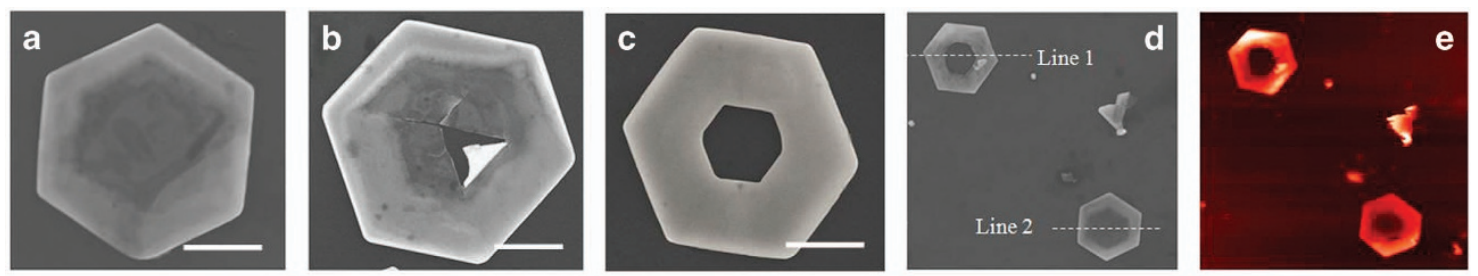

f
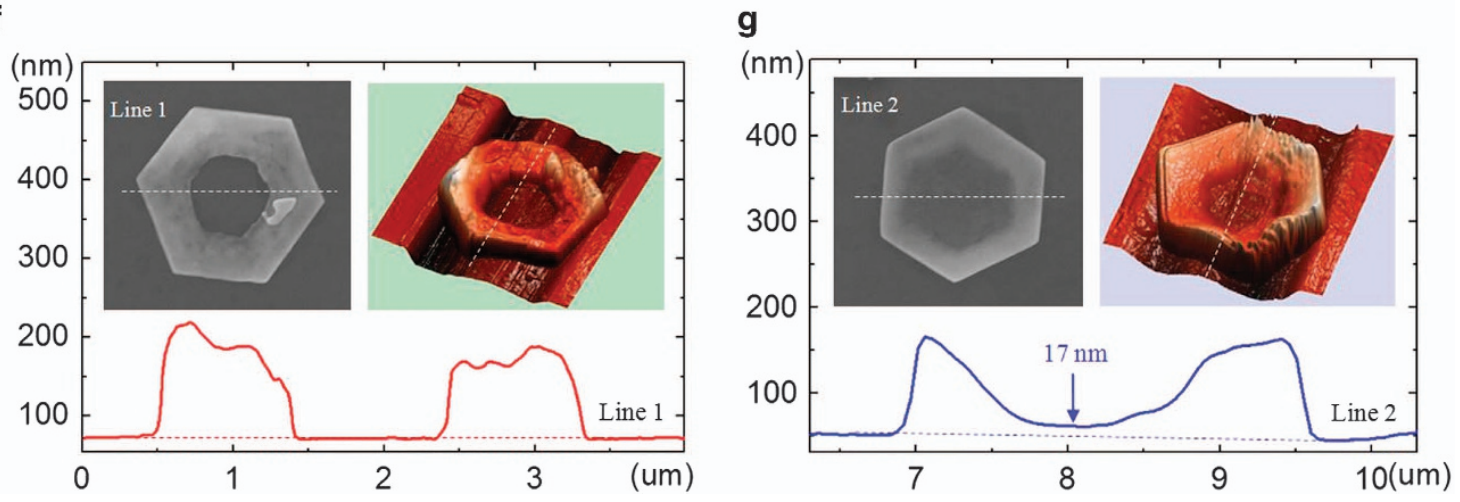

Figure 1 Typical morphologies of Au nanoplates and nanorings. (a-c) SEM images of the synthesized Au nanoplate and nanorings after 40 min for a, $1 \mathrm{~h}$ for b and $40 \mathrm{~h}$ for c. SEM image, matching atomic force microscopy pattern and corresponding cross-section profiles of Au nanoplates and nanorings after $40 \mathrm{~min}$ reaction for $\mathbf{d}, \mathbf{e}, \mathbf{f}$ and $\mathbf{g}$. The scale bars in $\mathbf{a}-\mathbf{c}$ are $1 \mu \mathrm{m}$. 
detected by a monochromator (Acton Spectra Pro 2150i) coupled to a CCD camera (Andor DR-328G-C01-SIL). The background signals measured in the area without any particles were subtracted from the scattering spectra, and then, the signals were calibrated by the spectral profile of the white light.

\section{RESULTS}

Au nanoplates and nanorings

The Au nanorings were synthesized through a stress-driven collapse and in situ etching processes within a facile reaction system consisting of $\mathrm{PEG} / \mathrm{HAuCl}_{4}$ aqueous solution (reaction 1). To tailor the central hole sizes, SDS was added into reaction 1; thus, the reaction system was composed of SDS/PEG/HAuCl 4 solution (reaction 2). Figure 1a, Supplementary Figure S1a and b show SEM images of the obtained Au nanoplates, which exhibit a hexagonal shape. Careful observation of the contrast between the central and edge regions of individual Au nanoplates reveals that hexagonal $\mathrm{Au}$ nanoplates are frame-like structures covered by a thin Au film in the central region. As the reaction proceeds, the thin film of the central region can crack spontaneously after $1 \mathrm{~h}$ of reaction (Figure $1 \mathrm{~b}$ and Supplementary
Figure S1c). By analyzing the crack traces shown in Figure 1b and Supplementary Figure S2, it is found that the cracks propagate along the diagonals of the hexagonal Au nanoplates. Unexpectedly, most of cracked thin films from the central regions of the $\mathrm{Au}$ nanoplates disappear and finally form hexagonal Au nanorings after $40 \mathrm{~h}$ (Figure $1 c$ and Supplementary Figure S1d). The SEM imagematching atomic force microscopy patterns (Figure 1d and e) and corresponding cross-section profiles (Figure if and g) show the geometrical dimensions of Au nanorings and nanoframes. According to the profiles shown in Figure If and g and Supplementary Figure S3, the height of the edge regions of the Au nanorings or nanoframes is $\sim 100-150 \mathrm{~nm}$. Notably, the Au nanoframes display a 'bowl'-like profile, and the heights of the films in the central regions of the nanoframes shown in Figure $1 \mathrm{~g}$ and Supplementary Figure S3 are $17 \mathrm{~nm}$ and $18 \mathrm{~nm}$, respectively. These observations reveal that the film in the central region is not symmetrically distributed and shifts to one side. Thus, it is reasonable to deduce that the Au nanoframes show a specific structure, that is, a grooved surface on one side and nearly flat a

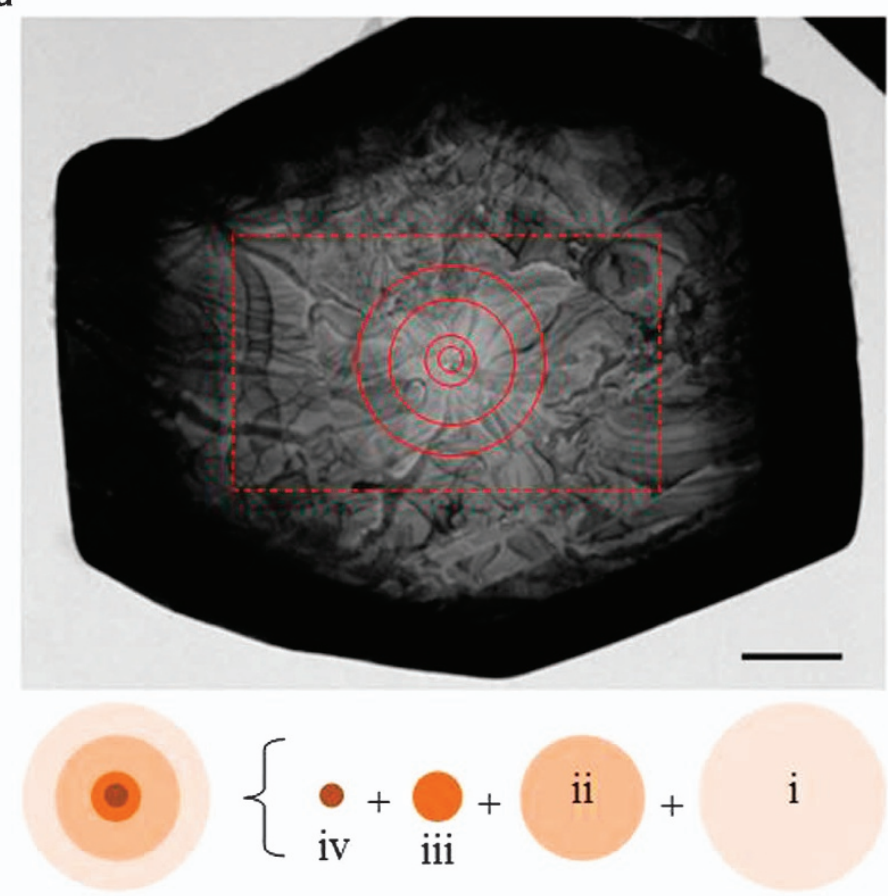

b
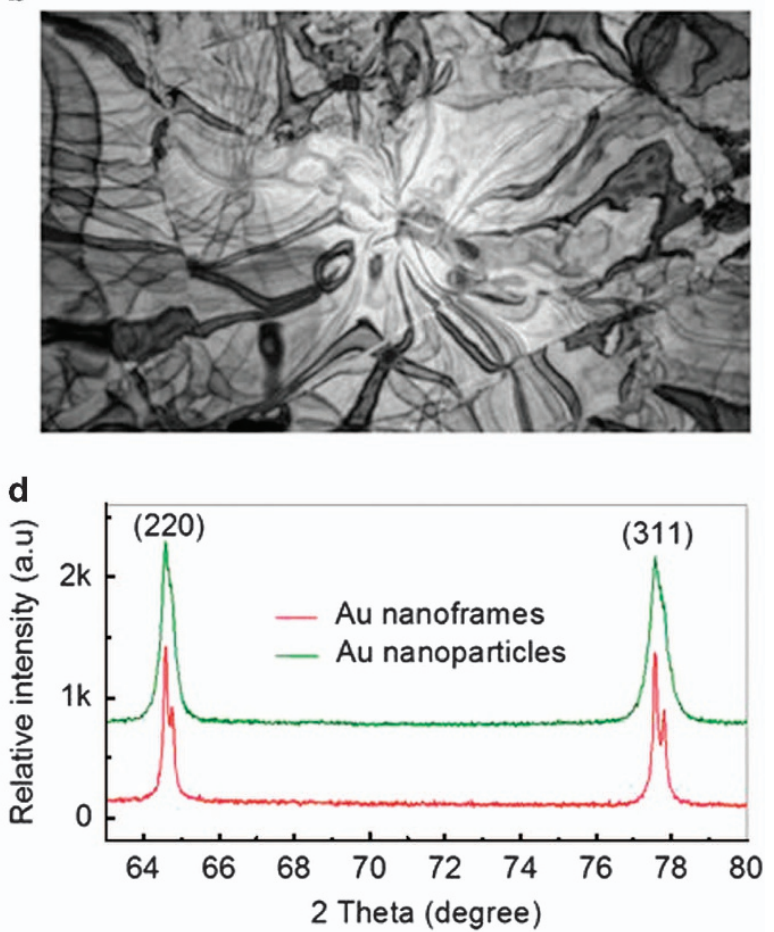

C
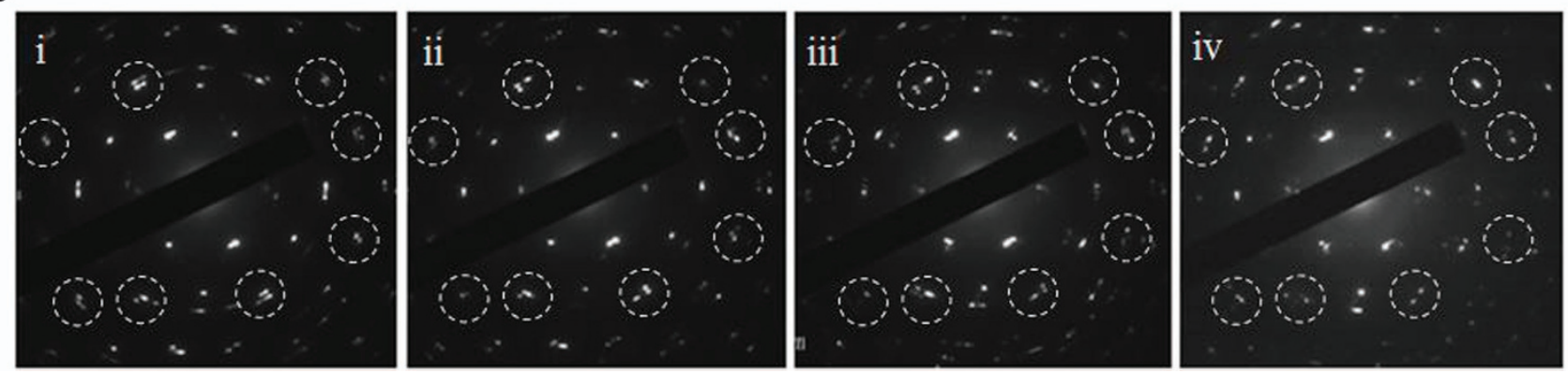

Figure 2 Stress characterization of the Au nanoframes. ( $\mathbf{a}$ and $\mathbf{b}$ ) TEM images and $\mathbf{c}$, selected area electron diffraction (SAED) patterns of the circled areas in a with sizes from i to iv. (d) XRD patterns of Au nanoframes and spherical nanoparticles that were synthesized in the same reaction system but with different conditions for comparison. The scale bar in a is $1 \mu \mathrm{m}$. 
surface on the other side (line-2). In fact, this type of structure has also been observed in the formation of $\mathrm{ZnO}$ nanorings. ${ }^{13}$

To investigate the shapes of the products under a variety of reaction conditions, we also studied the influences of reaction temperature and reactant concentrations. At relatively low reaction temperatures, for example, room temperature, the reaction is very slow, and no $\mathrm{Au}$ nanorings were observed. By contrast, at high temperatures, for example, $70^{\circ} \mathrm{C}$, a frame-like structure forms; however, within a relatively short reaction time, for example, $1.5-9 \mathrm{~h}$, the frame breaks up into fragments (Supplementary Figure S4). In addition, we also changed the concentrations of the PEG and $\mathrm{HAuCl}_{4}$. At low PEG concentrations, the frame-like structure is obtained (Supplementary Figure S5), but the central thin Au film cannot crack and form the ring-like structure. Instead, the seams of the frame-like structure release the stress by deformation. Increasing the concentration of $\mathrm{HAuCl}_{4}$ to 1.0 or $1.2 \mathrm{mM}$ (PEG constant), the products contain large spherical NPs (Supplementary Figure S6a and b). Interestingly, keeping the ratio of $\mathrm{Au}^{3+}: \mathrm{PEG}$ at $1: 2$ and the concentration of $\mathrm{Au}^{3+}$ ions at 0.75 and $1.0 \mathrm{mM}$, hexagonal nanoplates are formed, and a small pore can be observed in the center of each hexagonal Au nanoplate (Supplementary Figure S6c and d).

According to the above observations, the formation process of unique Au nanorings involves at least the following steps: nanoframe formation, break up of the central thin film and dissolution of the broken $\mathrm{Au}$ film (Figure 1c). Now, some basic questions are immediately raised and need to be addressed. For instance, how the frame-like structure forms, why the frame structure collapses, and how the cracked film dissolves and forms regular holes in the centers of the nanorings. In the following section, we shall address these questions in detail.

\section{Stress in Au nanoframes}

Figure 2a shows a TEM image of an individual hexagonal Au nanoframe. The region marked by the square box in Figure 2a is enlarged in Figure $2 b$, showing a beautiful band-like pattern. These bands or lines, which always start from the center and radiate down to the edges of the nanoframes, may be ascribed to bending of the thin crystals or the presence of multiple twinned structures. Similar continuous and randomly distributed pairs of lines can be seen across the faces of the flat crystals and have also been observed in $\mathrm{Au}$ nanocrystals synthesized using various approaches. ${ }^{30}$ Figure $2 \mathrm{c}$ shows a series of selected area electron diffraction (SAED) patterns taken from the selected regions marked in Figure 2a by circles (i, ii, iii and iv). Amazingly, the SAED patterns demonstrate many dimer-like spots along the diameter direction. Furthermore, these spots become weaker as the selected region decreases, that is, region iv. This unique SAED a

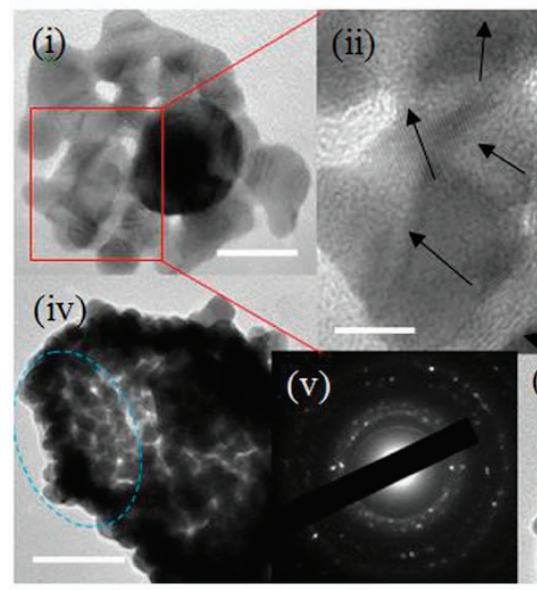

c

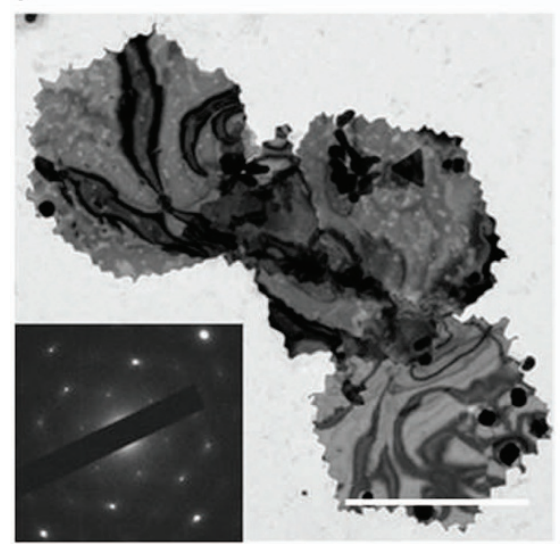

b

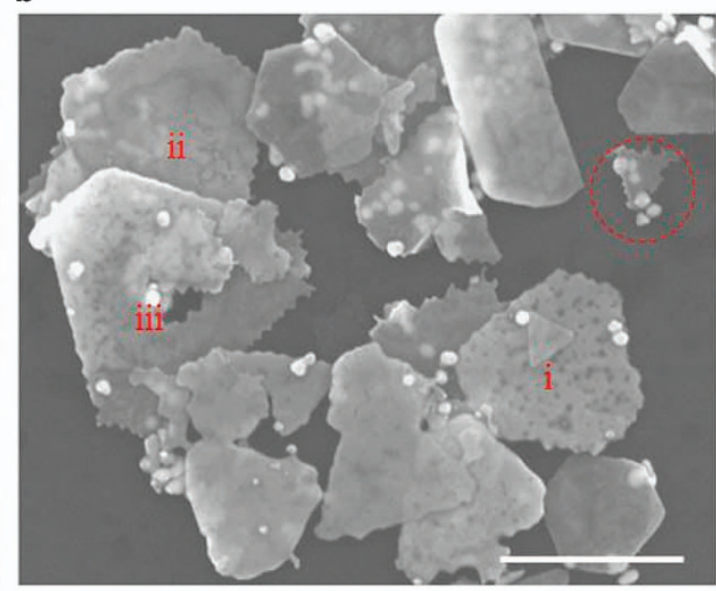

e

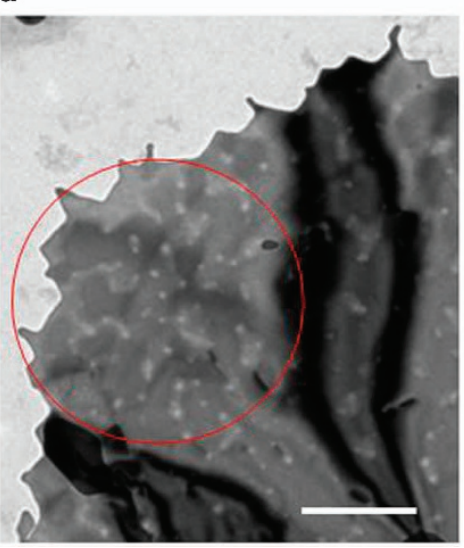

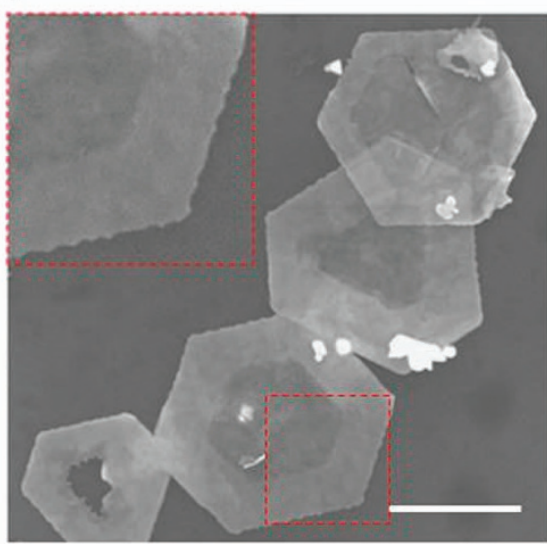

Figure 3 Analysis of the formation process of the Au nanoframes. Representative TEM and high-resolution transmission electron microscopy images of Au nanostructures formed after reaction times of a, $5 \mathrm{~min}, \mathbf{b}, \mathbf{c}$ and $\mathbf{d}, 30 \mathrm{~min}$, and e, $40 \mathrm{~min}$. The SAED pattern in Figure $3 a-v$ was recorded from the blue-circled region, and the SAED pattern in Figure $3 \mathrm{c}$ was recorded from the red-circled region in Figure $3 \mathrm{~d}$. Scale bars, a-i, $10 \mathrm{~nm}$, a-ii, $5 \mathrm{~nm}$, a-iii, $200 \mathrm{~nm}$, a-iv and a-vi, $50 \mathrm{~nm}, \mathrm{~b}, \mathrm{c}, \mathrm{e}, 1 \mu \mathrm{m}$, and d, $200 \mathrm{~nm}$. 
pattern has been rarely reported so far and indicates that an unusual stress exists in the hexagonal Au nanoframes.

To estimate the magnitude of such stresses, the XRD pattern of the Au nanoframes was measured. In addition, Au spherical NPs (Supplementary Figure S7) were synthesized using the same reaction system as the $\mathrm{Au}$ nanoframes but with different conditions for comparison. Figure $2 \mathrm{~d}$ shows the XRD patterns for the Au nanoframes and NPs, which are indexed to the diffraction from the (220) and (311) of fcc Au. The low-angle diffraction peaks, that is, (111) and (200), are shown in Supplementary Figure S8. Shoulder peaks can be observed from all diffraction planes for $\mathrm{Au} \mathrm{NPs}$, and for the $\mathrm{Au}$ nanoframes, the shoulder peaks become pronounced and split into two peaks, that is, $64.581^{\circ}$ and $64.771^{\circ}$ for (220) diffraction, and $77.567^{\circ}$ and $77.804^{\circ}$ for (311) diffraction, respectively. The XRD peak splitting commonly corresponds to the existence of stresses, as reported in various studies, such as ice freezing and multilayered film. ${ }^{31,32}$ According to the method reported by Varshney et al., the stress value is $\sim 200 \mathrm{MPa}^{33}$ In the current situation, the frame-like structure covered by a thin $\mathrm{Au}$ film may create an unusual stress because the thick frame may serve as a support to pin the deformation of the central $\mathrm{Au}$ layer. Therefore, the unique frame-like $\mathrm{Au}$ nanostructure is critical to the formation of $\mathrm{Au}$ nanorings by means of an unusual stress-driven structure collapse mechanism.

\section{Formation of Au nanoframes}

To illustrate how the frame-like structure forms, time-dependent intermediate specimens were characterized and are shown in Figure 3. After $5 \mathrm{~min}$ of reaction time, the products contain spherical seeds $(\sim 10 \mathrm{~nm})$, irregularly shaped aggregates of seeds (Figure 3a-i and iii), and polyhedral-like nanoplates (blue regions in Figure 3a-iv). The black arrows in the high-resolution transmission electron microscopy image shown Figure 3a-ii represent the crystallographic orientations of different grains, indicating that random aggregation is the polycrystalline essence. Meanwhile, the SAED pattern recorded from the blue-circled region shows that the polyhedral-like aggregates are still polycrystalline, displaying some separated diffraction spots. In addition, at this reaction stage, some relatively dense nanoplates can also be observed, for example, the TEM image in Figure 3a-vi, which shows obvious sintering and fusion features of aggregated NPs. This phenomenon seems to imply that these seed particles may fuse and be incorporated into the nanoplates as shown in Figure 3a-vi.

Typical morphologies of the products produced when the reaction proceeds for $\sim 30 \mathrm{~min}$ are shown in Figure $3 \mathrm{~b}-\mathrm{d}$. Figure $3 \mathrm{~b}$ shows the typical morphologies at this stage - a mixture consisting of plate-like aggregated NPs (i), nanoframes (ii), nanorings (iii) and some irregular nanoplates. The SAED pattern presented in Figure $3 \mathrm{c}$ recorded from the red-circled region of Figure $3 \mathrm{~d}$ shows the diffraction spots correspond to the Au fcc single-crystalline structure, revealing that, in the selected region, NP aggregates have converted into single crystalline structures. In addition, careful observation reveals that the edges of some nanoplates, for example, region ii in Figure $3 \mathrm{~b}$ and Supplementary Figure S9, have become thicker and denser relative to the central region of the nanoplates. This phenomenon becomes more obvious after a reaction time of $40 \mathrm{~min}$ as shown in Figure 3e. At this stage, the frame-like structure covered by a thin Au film in the central region has been formed (see the inset in Figure 3e).

\section{Growth mechanism of Au nanoframes and nanorings}

To gain insight into the transformation mechanism from the initial spherical $\mathrm{Au} \mathrm{NPs}$ to the final Au nanorings, we performed density functional theory calculations to determine the interactions between the PEG molecules and Au nanostructures (the details are provided in the supporting information). According to the density functional theory results, a 2D 'PEG-water' film (Figure $4 \mathrm{~b}$ ) may be created with the 'A-B-A' configuration (Figure 4a) via the formation of hydrogen bonds between water molecules and PEG molecules (Supplementary Figure S10). During the reduction of $\mathrm{Au}$ atoms, the produced $\mathrm{Au}$ atoms have higher adsorption energy with 'PEG-water' molecules a

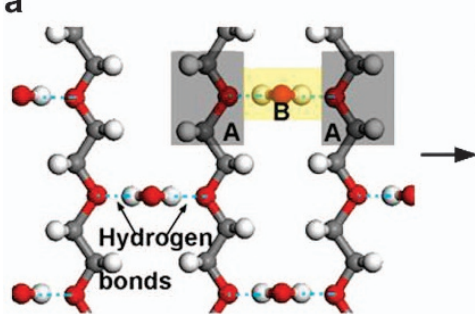

b

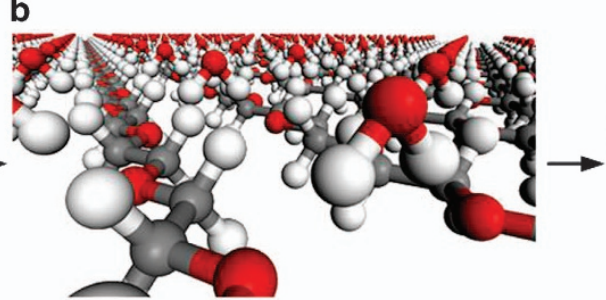

C

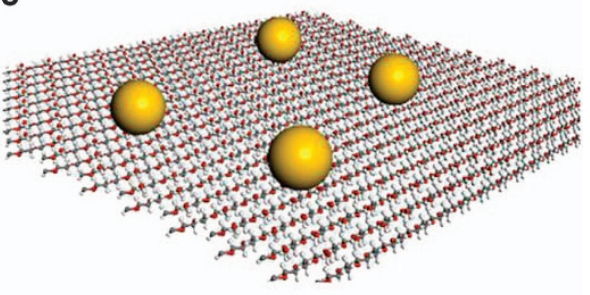

d
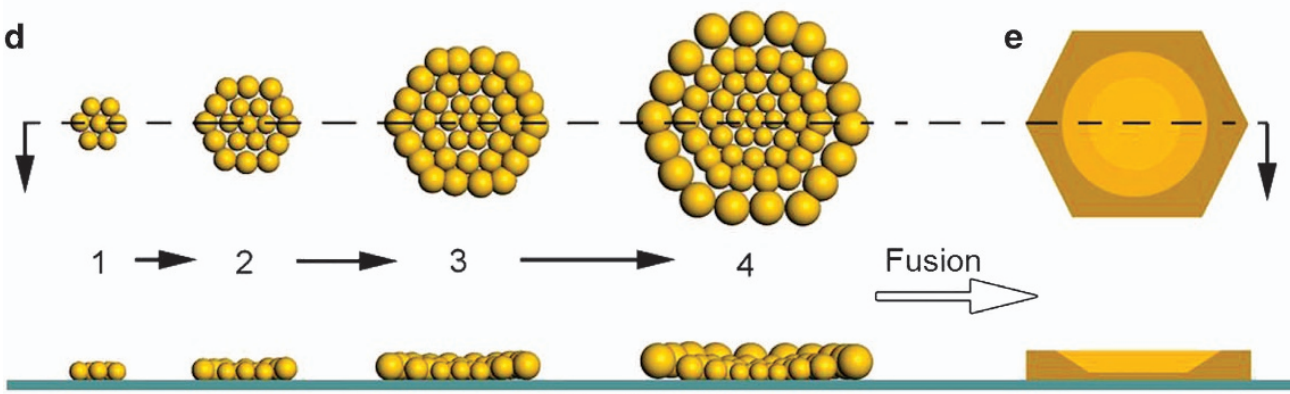

वरलिक्षि

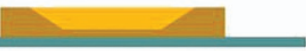

PEG+Water film

Figure 4 Schematic representation of the sequential steps leading to the formation of Au nanorings. (a and $\mathbf{b})$ Interactions between $\mathrm{H}_{2} \mathrm{O}$ molecules and PEG molecules via hydrogen bonds results in the formation of $2 \mathrm{D}$ assembly configurations. (c) Au nanoparticles absorb on the PEG/ $\mathrm{H}_{2} \mathrm{O}$ film. (d) The aggregation of $\mathrm{Au}$ nanoparticles on the $\mathrm{PEG} / \mathrm{H}_{2} \mathrm{O}$ film forms polycrystalline plates. (e) Recrystallization of these plates leads to single-crystalline Au nanoframes. (f) Stress-driven nanoframe breaking. (g) The formation of Au nanorings. 
a

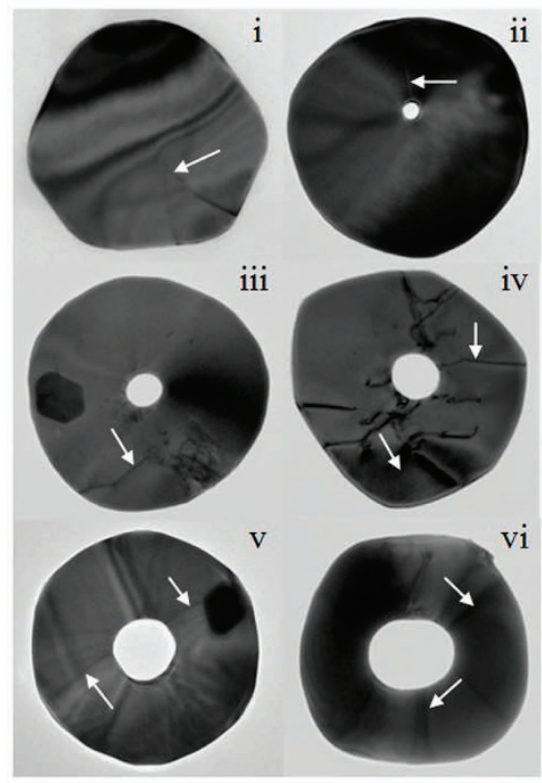

b

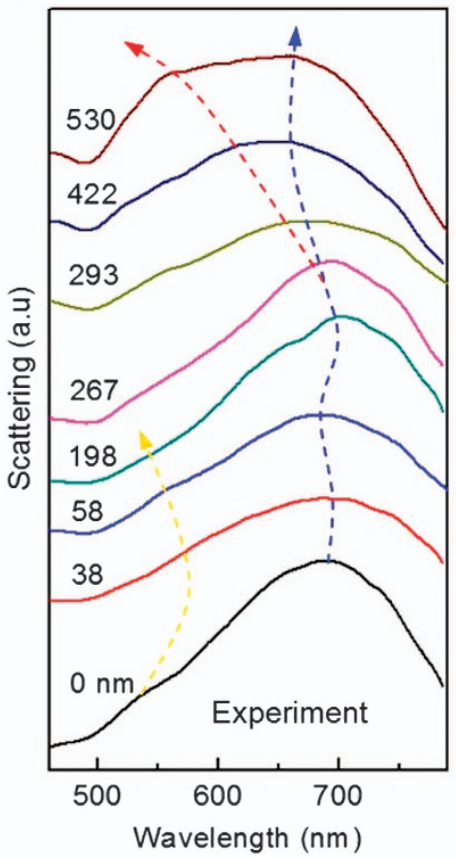

C

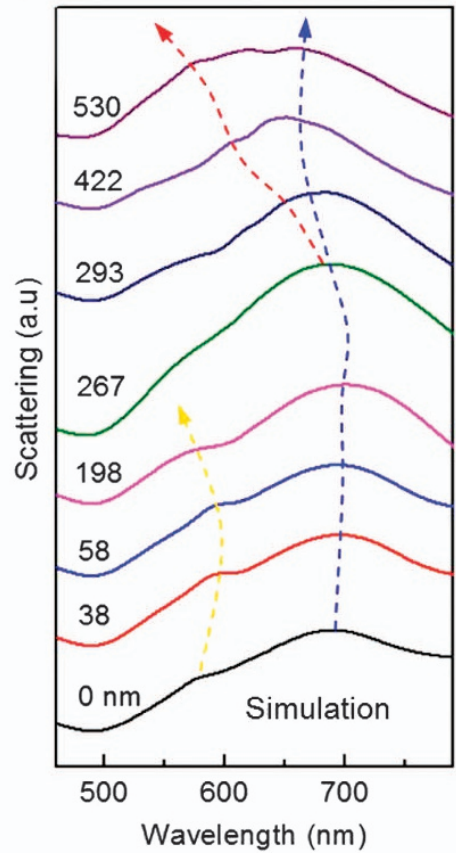

Figure 5 Representative TEM images of Au nanoplate and nanorings and optical characteristics of Au nanorings with various hole sizes. (a) Representative TEM images of Au nanoplate and nanorings. (b) Experimental and, (c) simulated scattering spectra from various hole sizes as indicated. The arrows in a reveal the presence of crystallographic defects.

than that with individual water molecules and PEG molecules (Supplementary Figure S11 and Supplementary Table S2). Thus, $\mathrm{Au}$ atoms preferentially adsorb and grow on the 2D 'PEG-water' film (Figure 4c).

According to the Lamer curve and classical nucleation theory, ${ }^{34-38} \mathrm{a}$ high supersaturation may be preferable to obtain small particle sizes. Thus, in the initial stage of nucleation, Au NPs with a small particle size are synthesized as shown in Figure 3a. Because the rate of $\left[\mathrm{AuCl}_{4}\right]^{-}$reduction in the current reaction system is relatively slow, as the supersaturation reduces, the resulting Au NPs show a relatively larger particle size (Figure 3a). This result is consistent with the TEM observations for the 2D Au NP aggregation shown in Figure 3a, in which the central particles display a relatively small size and the outer particles show a larger size (Figure 4d). In the following growth and coarsening stage, a recrystallization process of the NPs via sintering, fusion or ripening could occur to form dense single-crystalline Au nanoframes (Figure 3c,d and Figure 4e). In fact, similar to our observations, a NP fusion or Ostwald ripening process has been proposed to play an important role in 'aggregation-induced fast crystal growth'. ${ }^{39-41}$ The aggregated NPs may fuse others across a mismatched interface. Grain boundaries then migrate toward the adjacent particle, leading to the growth of one orientation at the expense of the other, which is quickly consumed. ${ }^{42}$

After the formation of $\mathrm{Au}$ nanoframes, a large stress around $\sim 200 \mathrm{MPa}$ estimated from XRD measurement then drives the structural breaking again. In this situation, in the thin central region, the stress imposed on each atom is relatively large, whereas in the thick edge region, each $\mathrm{Au}$ atom affords relatively small stress. Thus, the atoms in the central region can be etched easily. In addition, a relatively high chemical potential at the thin cracking sites may also be helpful to accelerate the etching process in these regions. Thus, $\mathrm{Au}$ nanorings are finally obtained (Figure $4 \mathrm{f}$ and $\mathrm{g}$ ). The proposed pathway for the formation of $\mathrm{Au}$ nanoframes and nanorings is summarized in Figure 4. However, we cannot exclude the potential role of Ostwald ripening or kinetically directed atom-by-atom dissolution. In reaction-2, we added the SDS into reaction-1 to magnify the etching function, and etching-driven $\mathrm{Au}$ nanoring formation was observed.

Figure $5 \mathrm{a}$ presents the typical morphologies of products synthesized via reaction 2, displaying various hole sizes of Au nanorings obtained by changing the reaction time (Supplementary Figure S12). The TEM images shown in Figure 4a indicate that the Au nanorings have a flat surface from the initial nanoplate (i) to the nanorings (ii-vi). Unlike the nanorings synthesized via nanoframe collapse (reaction 1), the $\mathrm{Au}$ nanorings obtained from reaction 2 have a different growth mechanism. The regions marked by arrows in Figure 4a confirmed the presence of crystallographic defects such as dislocations or linear defects, which are distributed radially with a higher concentration at the center of the nanoplate (Supplementary Figure S13). A high density of defects at the center may result in a higher local etching/reaction rate and eventually lead to the formation of a hole in the center. In reaction $2, \mathrm{SDS}, \mathrm{Cl}^{-}$or $\mathrm{O}_{2}$ may all serve as etching reagents to dissolve the defect sites and promote the formation of nanoholes, which could have similarity with the formation of nanorings in $\mathrm{CaCO}_{3}, \mathrm{CuO}, \mathrm{Zn}-\mathrm{Al}$ and so on. ${ }^{43-45} \mathrm{In}$ fact, it has been found that surfactant, that is, SDS, SDBS, $\mathrm{PEG}, \mathrm{AOT}^{-}$, $\mathrm{PEDTA}$, may play an important role in the formation of ring-like structures in a variety of chemical reactions. ${ }^{46-49}$

\section{Optical characteristics of Au nanorings with tunable hole sizes}

The current direct solution-based chemical synthetic strategy to prepare $\mathrm{Au}$ nanorings not only displays a novel growth mechanism but also provides control over the hole sizes of the Au nanorings via reaction 2. The optical properties of Au nanorings with diverse hole sizes were investigated by single-particle scattering spectroscopy. A pattern-matching method was employed to correlate the precise 
a

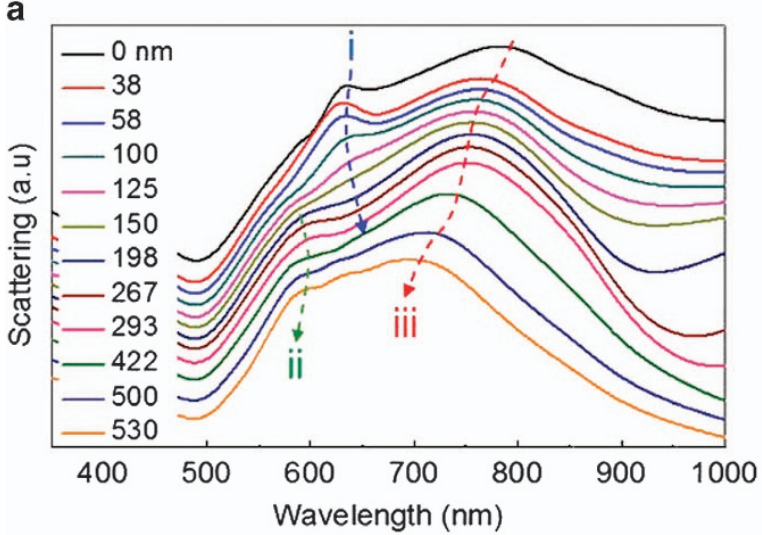

b

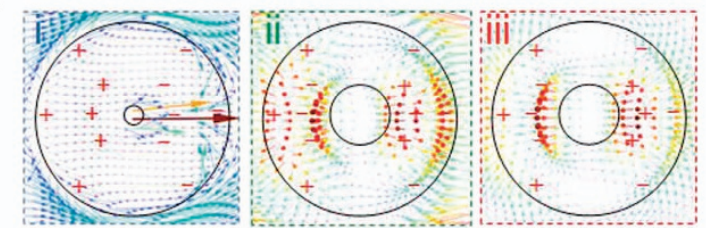

d

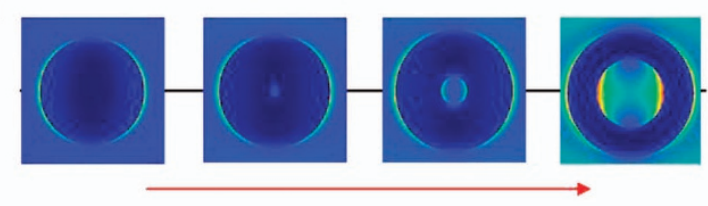

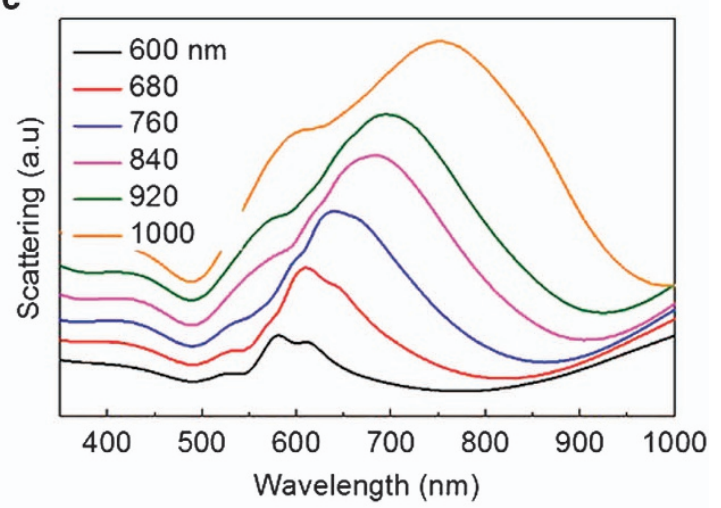

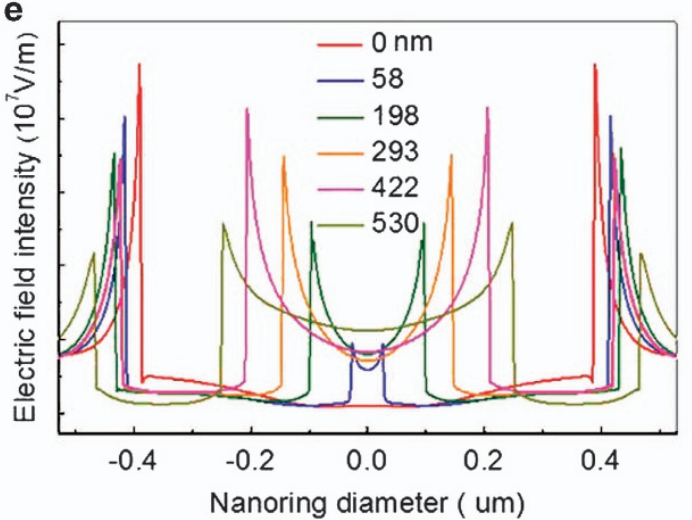

Figure 6 Analysis of the optical characteristics of Au nanorings with various hole sizes. (a) Scattering spectra as a function of hole size with a constant outer diameter of $1 \mu \mathrm{m}$. (b) The charge distributions and near-field vectors for nanorings with hole sizes of 58 and $293 \mathrm{~nm}$ at a constant outer diameter of $1 \mu \mathrm{m}$ under excitation at 634 (i), 604 (ii) and $740 \mathrm{~nm}$ (iii). (c) The scattering spectra for various outer diameters and a constant hole size of $293 \mathrm{~nm}$. (d) The electric-field intensity distributions for various hole sizes, and $\mathbf{e}$, cross sections of the electric field intensity distributions along the solid black line across the Au nanoplates and nanorings.

nanostructures of $\mathrm{Au}$ nanorings and their spectra. The SEM images and the corresponding scattering spectra of Au nanorings on an ITO coated glass substrate are shown in Figure 5 and Supplementary Figure S14, respectively. Figure $5 \mathrm{~b}$ and $\mathrm{c}$ are the experimental and corresponding simulated scattering spectra obtained using the finitedifference time-domain (FDTD) method, which show reasonable agreement. Notably, the Au nanorings display tunable localized surface plasmon resonances in the visible to the near infrared. In the case of small hole sizes, for example, 0,38 or $58 \mathrm{~nm}$, the scattering spectra exhibit a broader feature with a weak shoulder in the $550-600 \mathrm{~nm}$ region. With increasing hole size, for example, 198, 267 and $293 \mathrm{~nm}$, the spectra gradually narrow. When the hole sizes increases to more than $293 \mathrm{~nm}$, for example, 422 and $530 \mathrm{~nm}$, the spectra blue shift and broaden again.

To explain the surface plasmon resonance (SPR) modes of Au nanorings, we systematically examined the simulated scattering spectra for diverse hole sizes and a constant outer diameter of $1 \mu \mathrm{m}$ (Figure 6a). As the hole size increases, the peaks (iii) initially located at $780 \mathrm{~nm}$ blue shift to $\sim 700 \mathrm{~nm}$, and this shifting is more obvious when the hole sizes are larger than $293 \mathrm{~nm}$. This result is consistent with the spectroscopy feature obtained from experiments and shown in Figure 5b. At small hole sizes, for example, $<100 \mathrm{~nm}$, a shoulder peak (peak i) can always be observed in the region of $\sim 650 \mathrm{~nm}$, and it gradually disappears as the hole size increases. When the hole size is larger than $\sim 198 \mathrm{~nm}$, another shoulder peak (peak ii, $\sim 580 \mathrm{~nm}$ ) appears again. From the charge distribution and near-field vector shown in Figure 6b, the Au nanorings demonstrate obvious hybridization of surface plasmons. With small hole sizes, for example, $58 \mathrm{~nm}$, peak $\mathrm{i}$ and peak iii may be identified as the bonding modes and antibonding modes, which result from the nanoholes and outer edges of the Au nanorings, respectively (Supplementary Figure S15). When the hole sizes become even larger, for example, $293 \mathrm{~nm}$, the SPR modes for peaks ii and iii are both antibonding modes with the contributions from interacting inner hole and outer edge (Supplementary Figure S16). In fact, similar localized surface plasmon resonance features have also been observed in various ring-like Au nanostructures fabricated by nanosphere lithography and electron beam lithography techniques. ${ }^{50,51}$ In addition, we also studied the influences of various outer diameters on the SPR modes with constant inner hole sizes, for example, 58 and $293 \mathrm{~nm}$. Figure $6 \mathrm{c}$ and Supplementary Figure S17 indicate that, under a constant inner hole size, the peaks redshift as the outer diameter of the Au nanorings increases. The influence of the inner hole size on the SPR features can also be revealed by the electric field intensity distributions (Figure $6 \mathrm{~d}$ and e). According to the $\left|E_{\text {outer }}\right| /\left|E_{\text {inner }}\right|$ ratio, the value is $8(4 / 0.5)$ at a hole size of $38 \mathrm{~nm}$ and equal to $0.84(2.1 / 2.5)$ when the hole size is $530 \mathrm{~nm}$. Thus, as the hole size increases, the strong near-fields initially concentrated around the outer edge region move to the inner hole region, as shown in Figure 6d and e. Therefore, the Au nanorings display wide tunability for both spectroscopy and near-field distribution, which are highly attractive for many applications, such as chemical and biological sensors, NP trapping and field-enhanced spectroscopy. 


\section{DISCUSSION}

In this work, for the first time, we report a new stress-driven structure collapse and in situ etching mechanism for the synthesis of $\mathrm{Au}$ nanorings via a direct one-pot solution-based chemical reaction. Two types of $\mathrm{Au}$ nanorings are obtained (Figures $1 \mathrm{c}$ and $5 \mathrm{a}$ ) using facile reaction systems consisting of aqueous $\mathrm{PEG} / \mathrm{HAuCl}_{4}$ (reaction 1) and $\mathrm{SDS} / \mathrm{PEG} / \mathrm{HAuCl}_{4}$ (reaction 2) solutions. In reaction 1 , the unique NP aggregation style is believed to play an important role in the formation of Au nanoframes, which display novel structural features with thick edges relative to the central regions of the aggregates. The following recrystallization process via NP sintering, fusion or ripening results in the formation of Au nanoframes. An unusual stress of $\sim 200 \mathrm{MPa}$ may be created within this novel frame-like structure, thus resulting in structural breakup and formation of Au nanorings. In reaction 2, the simple addition of SDS, which serves as the etching reagent, contributes to the transformation of the $\mathrm{Au}$ nanostructures from initial nanoplates to nanorings. The resulting Au nanorings display unique structural characteristics, that is, tunable hole sizes from $\sim 10 \mathrm{~nm}$ to hundreds of nanometers with a constant nanoring size of $\sim 1 \mu \mathrm{m}$. The mechanism reported here is conceptually different from earlier work of $\mathrm{Au}$ nanorings obtained from, for example, galvanic reaction routes, which cannot produce novel structures with tunable inner hole sizes at such a wide level. We envision that our stress-driven structure collapse and etching mechanism may open up new perspectives to create a novel class of ring-like nanomaterials with uniform and tunable geometric dimensions through the optimization of the etching reagents, reaction time and surfactants.

\section{CONCLUSION}

In summary, the $\mathrm{Au}$ nanorings synthesized via the mechanism reported here represent a new platform to fundamentally comprehend the dependence of plasmonic characteristics on central hole size, that is, from hundreds down to tens of nanometers. The optical responses of the $\mathrm{Au}$ nanorings investigated using single-particle scattering spectroscopy and FDTD calculation exhibit tunable localized surface plasmon resonance features as a function of hole size. The experimental scattering spectra are consistent with the numerical calculations and interpreted as originating from the near-field coupling of the bonding and antibonding modes on the inner and outer surfaces of the nanorings. We hope that the unique synthetic strategy may open new opportunities to obtain ring-like nanomaterials that are suitable for a variety of practical applications related to optical sensors, catalysis, medicine, surface-enhanced Raman scattering and second harmonic generation among others.

\section{ACKNOWLEDGEMENTS}

This work was supported by the National Natural Science Foundation of China (Nos. 21675122, 51171139, 11304188, and 11434017), Scientific New Star Program in Shann Xi Province (No. 2012KJXX-03), the Fundamental Research Funds for the Central Universities (No. xkjc2014004) and the specialized research fund for the doctoral program of higher education of China (20130201110032). We thank Prof. H. Y. Chen and B. Ren for helpful discussions.

Author contributions: J Li synthesized the materials and carried out the characterizations of the structures. C Tian and Q Gao completed the atomic force microscopy measurements. C Tian and X Wang conducted the FDTD calculations. N Gao and X Wen carried out the measurement of the SEM matched scattering spectra. C Ma assisted with the TEM characterization. $\mathrm{H}$ You conducted the density functional theory calculations. The group of Z Yang, Q H Xu and Q Xiong conducted the FDTD calculations and spectroscopy measurements. J Fang designed and supervised the project and wrote the manuscript. All authors discussed the results and commented on the manuscript.

1 Kim, Y., Li, W., Shin, Y. \& Lee, M. Development of toroidal nanostructures by self-assembly: rational designs and applications. Acc. Chem. Res. 46, 2888-2897 (2013).

$2 \mathrm{Hu}, \mathrm{X}$. L., Yu, J. C., Gong, J. M., Li, Q \& Li, G. S. a-Fe $\mathrm{O}_{3}$ nanorings prepared by a microwave-assisted hydrothermal process and their sensing properties. Adv. Mater. 19, 2324-2329 (2007)

3 Jia, C. J., Sun, L. D., Luo, F., Han, X. D., Heyderman, L. J., Yan, Z. G., Yan, C. H., Zhang, K., Zhang, Z., Takano, M., Hayashi, N., Eltschka, M., Klaui, M., Rudiger, U., Kasama, T., Cervera-Gontard, L. C., Dunin-Borkowshi, R. E., Tzvetkov, G. \& Jorg, R. Large-scale synthesis of single-crystalline iron oxide magnetic nanorings. J. Am. Chem. Soc. 130, 16968-16977 (2008).

4 Lorente-Crespo, M., Wang, L., Ortuno, R., Garica-Meca, C., Ekinci, Y. \& Martinez, A. Magnetic hot spots in closely spaced thick gold nanorings. Nano Lett. 13, 2654-2661 (2013)

5 Liusman, C., Li, S. Z., Chen, X. D., Wei, W., Zhang, H., Schatz, G. C., Boey, F. \& Mirkin, C. A. Free-standing bimetallic nanorings and nanoring arrays made by on-wire lithography. ACS Nano 4, 7676-7682 (2010).

6 Aizpurua, J., Hanarp, P., Sutherland, D. S., Kall, M., Bryant, G. W. \& Gracia de Abajo, F. J. Optical properties of gold nanorings. Phys. Rev. Lett. 90, 057401 (2003).

7 Larsson, E. M., Alegret, J., Kall, M. \& Sutherland, D. S. Sensing characteristics of NIR localized surface Plasmon resonances in gold nanorings for application as ultrasensitive biosensors. Nano Lett. 7, 1256-1263 (2007).

$8 \mathrm{Hu}$, Y., Chou, T., Wang, H. \& Du, H. Monodisperse colloidal gold nanorings: synthesis and utility for surface-enhanced Raman scattering. J. Phys. Chem. C 118, 16011-16018 (2014).

9 Lehr, D., Reinhold, J., Thiele, I., Hartung, H., Dietrich, K., Menzel, C., Pertsch, T., Kley, E. B. \& Tunnermann, A. Enhancing second harmonic generation in gold nanoring resonators filled with lithium niobate. Nano Lett. 15, 1025-1039 (2015).

10 Shahjamali, M. M., Bosman, M., Cao, S. W., Huang, X., Cao, X. H., Zhang, H., Pramana, S. S. \& Xue, C. Surfactant-free sub-2 $\mathrm{nm}$ ultrathin triangular gold nanoframes. Small 9, 2880-2886 (2013).

11 Mclellan, J. M, Geissler, M. \& Xia, Y. N. Edge spreading lithography and its application to the fabrication of mesoscopic gold and silver rings. J. Am. Chem. Soc. 126, 10830-10831 (2004).

12 Metraux, G. S., Cao, Y. C., Jin, R. C. \& Mirkin, C. A. Triangular nanoframes made of gold and silver. Nano Lett. 3, 519-522 (2003).

13 Li, F., Ding, Y., Gao, P. X., Xin, X. Q. \& Wang, Z. L. Single-crystal hexagonal disks and rings of $\mathrm{ZnO}$ : low temperature large-scale synthesis and growth mechanism. Angew. Chem. 116, 5350-5354 (2004).

14 Jia, C. J., Sun, L. D., Yang, Z. G., You, L. P., Luo, F., Han, X. D., Pang, Y. C., Zhang, Z. \& Yan, C. H. Single-crystalline iron oxide nanotubes. Angew. Chem. Int. Ed. 44, 4328-4333 (2005).

15 Liang, Y. J., Wang, W. Z., Zeng, B. Q., Zhang, G. L., He, Q. Y. \& Fu, J. L. Influence of $\mathrm{NaOH}$ on the formation and morphology of $\mathrm{Bi}_{2} \mathrm{Te}_{3}$ nanostructures in a solvothermal process: from hexagonal nanoplates to nanorings. Mater. Chem. Phys. 129, 90-98 (2011).

16 Meldrum, F. C. \& Colfen, H. Controlling mineral morphologies and structures in biological and synthetic systems. Chem. Rev. 108, 4332-4432 (2008).

17 Peng, Y., Xu, A. W., Deng, B., Antonietti, M. \& Colfen, H. Polymer-controlled crystallization of zinc oxide hexagonal nanorings and disks. J. Phys. Chem. B 110, 2988-2993 (2006)

18 Xu, J., Wang, H., Liu, C. C., Yang, Y. M., Wang, Y. W., Wang, F., Liu, X. G., Xing, B. G. \& Chen, H. Y. Mechanical nanosprings: induced coiling and uncoiling of ultrathin $\mathrm{Au}$ nanowires. J. Am. Chem. Soc. 132, 11920-11922 (2010).

19 Wang, P. P., Yang, Y., Zhuang, J. \& Wang, X. Self-adjustable crystalline inorganic nanocoils. J. Am. Chem. Soc. 135, 6834-6837 (2013).

20 Wei, Q. L., Tan, S. S., Liu, X. Y., Yan, M. Y., Wang, F. C., Li, Q. D., An, Q. Y., Sun, R. M., Zhao, K. N., Wu, H. G. \& Mai, L. Q. Novel polygonal vanadium oxide nanoscrolls as stable cathode for lithium storage. Adv. Funct. Mater. 25, 1773-1779 (2015).

21 Han, J. H., Lee, S., Yoo, D., Lee, J., Jeong, S., Kim, J. \& Cheon, J. Unveiling chemical reactivity and structural transformation of two-dimensional layered nanocrystals. J. Am. Chem. Soc. 135, 3736-3739 (2013)

22 Jeong, S., Han, J. H., Jang, J., Seo, J., Kim, J. \& Cheon, J. Transformative two-dimensional layered nanocrystals. J. Am. Chem. Soc. 133, 14500-14504 (2011).

23 Hu, P., Pramana, S. S., Cao, S. W., Ngaw, C. K., Lin, J. D., Loo, S. C. J. \& Tan, T. T. Y. lon-induced synthesis of uniform single-crystalline sulphie-based quaternary-alloy hexagonal nanorings for highly efficient photocatalytic hydrogen evolution. Adv. Mater. 25, 2567-2572 (2013).

24 Liu, B. \& Zeng, H. C. Semiconductor rings fabricated by self-assembly of nanocrystals. J. Am. Chem. Soc. 127, 18262-18268 (2005).

$25 \mathrm{Hu}$, M. J., Lu, Y., Zhang, Sen., Guo, S. R., Lin, B., Zhang, M. \& Yu, S. H. High yield synthesis of bracelet-like hydrophilic $\mathrm{Ni}$-Co magnetic alloy flux-closure nanorings. J. Am. Chem. Soc. 130, 11606-11607 (2008).

26 Cho, K. S., Talapin, D. V., Gaschler, W. \& Murray, C. B. Designing PbSe nanowires and nanorings through oriented attachment of nanoparticles. J. Am. Chem. Soc. 127, 7140-7147 (2005) 
27 Zinchenko, A. A., Yoshikawa, K. \& Baigl, D. DNA-templated silver nanorings. Adv. Mater. 17, 2820-2823 (2005).

28 Preston, T. C. \& Signorell, R. Formaton of gold particles on nanoscale toroidal DNA assembled with bis (ethylenediamine) gold (III). Langmuir 26, 10250-10253 (2010).

29 Wang, C. R., Fang, Y. \& Feng, J. W. Synthesis of Au nanorings using SDS-PEG cluster as the soft template under mild conditions. Acta Chim. Sin. 65 (12), 1177-1180 (2007).

30 Shao, Y., Jin, Y. D. \& Dong, S. J. Synthesis of gold nanoplates by aspartate reduction of gold chloride. Chem. Commun. 10, 1104-1105 (2004).

31 Somayazulu, M., Shu, J., Zha, C., Goncharov, A. F., Tschauner, O., Mao, H. \& Hemley, R. J. In situ high-pressure $x$-ray diffraction study of $\mathrm{H}_{2} \mathrm{O}$ ice Vii. J. Chem. Phys. 128, 064510 (2008).

32 Li, J., Wu, X. L., Hu, D. S., Yang, Y. M., Qiu, T. \& Shen, J. C. Splitting of x-ray diffraction peak in $\left(\mathrm{Ge}: \mathrm{SiO}_{2}\right) / \mathrm{SiO}_{2}$ multilayers. Solid State Commun. 131, 21-25 (2004).

33 Varshney, D. B., Elliott, J. A., Gatlin, L. A., Kumar, S., Suryanarayanan, R. \& Shalaev, E. Y. Synchrotron x-ray diffraction invstigation of the anomalous behavior of ice during freezing of aqueous systems. J. Phys. Chem. B 113, 6177-6182 (2009).

34 You, H. J. \& Fang, J. X. Particle-mediated nucleation and growth of solution-synthesized metal nanocrystals: a new story beyond the LaMer curve. Nano Today 11, 145-167 (2016).

35 Fang, J. X., Du, S. Y., Lebedkin, S., Li, Z. Y., Kruk, R., Kappes, M. \& Hahn, H. Gold mesostructures with tailored surface tophography and their self-assembly arrays for surface-enhanced Raman spectroscopy. Nano Lett. 10, 5006-5013 (2010).

36 Fang, J. X., Leufke, P. M., Kruk, R., Wang, D., Scherer, T. \& Hahn, H. External electric field driven 3D ordering architecture of silver (i) oxide mesosuperstructures. Nano Today 5, 175-182 (2010).

37 Fang, J. X., Ding, B. J. \& Song, X. P. Self-assembly mechanism of platelike silver mesocrystal. Appl. Phys. Lett. 91, 083108 (2007).

38 Fang, J. X., Ma, X. N., Cai, H. H., Song, X. P. \& Ding, B. J. Nanoparticle-aggregated 3D monocrystalline gold dendritic nanostructures. Nanotechnology 17, 5841-5845 (2006).

39 Zhuang, Z. Y., Huang, F., Lin, Z. \& Zhang, H. Z. Aggregation-induced fast crystal growth of SnO2 nanocrystals. J. Am. Chem. Soc. 134, 16228-16234 (2012).

40 Shankar, S. S., Rai, A., Ankamwar, B., Singh, A., Ahmad, A. \& Sastry, M. Biological synthesis of triangular gold nanoprisms. Nat. Mater. 3, 482-488 (2004).

41 Tangeysh, B., Tibbetts, K. M., Odhner, J. J., Wayland, B. B. \& Levis, R. J. Triangular gold nanoplate growth by oriented attachment of Au seeds generated by strong field laser reduction. Nano Lett. 15, 3377-3382 (2015).
42 Li, D. S., Nielsen, M. H., Lee, J. R. I., Frandsen, C., Banfield, J. F. \& de Yoreo, J. J. Direction-specific interactions control crystal growth by oriented attachment. Science 336, 1014-1018 (2012)

43 Qi, L. M., Li, J. \& Ma, J. M. Biomimetic morphogenesis of calcium carbonate in mixed solutions of surfactants and double-hydrophilic block copolymers. Adv. Mater. 14, 300-303 (2002).

44 Wang, X. Q., Xi, G. C., Xiong, S. L., Liu, Y. K., Xi, B. J., Yu, W. C. \& Qian, Y. T. Solution-phase synthesis of single-crystal $\mathrm{CuO}$ nanoribbons and nanorings. Cryst. Growth Des. 7, 930-934 (2007).

45 Yilmaz, C., Unal, U. \& Acar, H. Y. Platelets to rings: influence of sodium dodecyl sulfate on Zn-Al layered double hydroxide morphology. J. Solid State Chem. 187, 295-299 (2012).

46 Walsh, D., Lebeau, B \& Mann, S. Morphosynthesis of calcium carbonate (vaterite) microsponse. Adv. Mater. 11, 324-328 (1999).

47 Ren, L., Cao, M. H., Shi, S. F. \& Hu, C. W. Vanadium oxide nanodisks: synthesis, characterization, and electrochemical properties. Mater. Res. Bull. 47, 85-91 (2012).

48 Zhou, Y. \& Zeng, H. C. Simultaneous synthesis and assembly of noble metal nanoclusters with variable micellar templates. J. Am. Chem. Soc. 136, 13805-13817 (2014).

49 Tan, L. H., Xing, S. X., Chen, T., Chen, G., Huang, X., Zhang, H. \& Chen, H. Y. Fabrications of polymer nanocavities with tailored openrings. ACS Nano 3 3469-3474 (2009).

50 Ye, J., Dorpe, P. V., Lagae, L., Maes, G. \& Borghs, G. Observation of plasmonic dipolar anti-bonding mode in silver nanoring structures. Nanotechnology 20, 465203 (2009).

51 Cai, Y. J., Li, Y., Nordlander, P. \& Cremer, P. S. Fabrication of elliptical nanorings with highly tunable and multiple plasmonic resonances. Nano Lett. 12, 4881-4888 (2012).

(c) (i) This work is licensed under a Creative Commons Attribution 4.0 International License. The images or other third party material in this article are included in the article's Creative Commons license, unless indicated otherwise in the credit line; if the material is not included under the Creative Commons license, users will need to obtain permission from the license holder to reproduce the material. To view a copy of this license, visit http:// creativecommons.org/licenses/by/4.0/

(C) The Author(s) 2016

Supplementary Information accompanies the paper on the NPG Asia Materials website (http://www.nature.com/am). 